\title{
Characterizations of Fuzzy Ideals in Coresiduated Lattices
}

\author{
Yan Liu ${ }^{1}$ and Mucong Zheng ${ }^{2}$ \\ ${ }^{1}$ College of Science, Xian University of Science and Technology, Xian 710054, China \\ ${ }^{2}$ School of Mathematics and Information Science, Shaanxi Normal University, Xi'an 710119, China \\ Correspondence should be addressed to Mucong Zheng; zhengmucong@aliyun.com
}

Received 27 January 2016; Revised 30 June 2016; Accepted 3 July 2016

Academic Editor: Remi Léandre

Copyright (c) 2016 Y. Liu and M. Zheng. This is an open access article distributed under the Creative Commons Attribution License, which permits unrestricted use, distribution, and reproduction in any medium, provided the original work is properly cited.

\begin{abstract}
The notions of fuzzy ideals are introduced in coresiduated lattices. The characterizations of fuzzy ideals, fuzzy prime ideals, and fuzzy strong prime ideals in coresiduated lattices are investigated and the relations between ideals and fuzzy ideals are established. Moreover, the equivalence of fuzzy prime ideals and fuzzy strong prime ideals is proved in prelinear coresiduated lattices. Furthermore, the conditions under which a fuzzy prime ideal is derived from a fuzzy ideal are presented in prelinear coresiduated lattices.
\end{abstract}

\section{Introduction}

Residuated lattices provide an algebra frame for the algebraic semantics of formal fuzzy logics such as MV-algebras, BLalgebras, and $R_{0}$-algebras (or MTL-algebras) [1-6]. Filters play crucial important role in the proof of the completeness of these logics. Many results about the filter theory of various fuzzy logical algebras have sprang out [7-11]. In fact, ideal and filter are considered as the dual notions of the logical algebra systems in the view of algebra structure. Ideal is also interesting because it is closely related to congruence relation. It plays a vital role in Chang's Subdirect Representation Theorem of MV-algebras [3]. Actually, the original definition of MV-algebra was characterized by the operator $\oplus$ which could be looked as the generation of conorm in lattices. Obviously, there existed an operator $\theta$ such that $(\oplus, \ominus)$ constituted a pair just like adjoint pair $(\otimes, \rightarrow$ ). Therefore, the definition of coadjoint pair was presented in [12], and the definition of coresiduated lattice, as the dual algebra structure of residuated lattice, was introduced in [12]. Coresiduated lattices provide a new frame for logic algebras. It was masterly used to obtain the unified form of intuitionistic fuzzy implications and the Triple I method solutions of intuitionistic fuzzy reasoning problems in [13]. It was also used to obtain the unified form of intuitionistic fuzzy difference operators and the Triple D method solutions of intuitionistic fuzzy reasoning problems in [14]. The properties of ideals in coresiduated lattices were investigated and the embedding theorem of coresiduated lattices was obtained in [15].

In recent years, fuzzy filters in various logic algebras have captured many scholars' attention. Liu and Li proposed the notion of fuzzy filters to BL-algebras and obtained their relative properties $[16,17]$. Jun et al. investigated fuzzy filters in MTL-algebras and lattice implication algebras [18]. Zhu and Xu extended the fuzzy filters in BL-algebras and MTLalgebras to general residuated lattices [19].

In this paper, we intend to introduce the notions of fuzzy ideals in coresiduated lattices and develop the ideal theory of coresiduated lattices. The rest of the paper is structured as follows. In Section 2 we recall the basic notions and existing results that will be used in the paper. In Section 3 we introduce the concept of fuzzy ideals in coresiduated lattices and present the characterizations of fuzzy ideals. In Section 4 we introduce the concepts of fuzzy prime ideals and fuzzy strong prime ideals in coresiduated lattices and obtain some of their characterizations. In Section 5 we advance the fuzzy prime ideal and fuzzy strong prime ideal in prelinear coresiduated lattices.

\section{Preliminaries}

In this section, we recall some basic concepts and results, which we will need in the subsequent sections. 
Definition 1 (see [12]). Let $P$ be a poset. $(\oplus, \ominus)$ is called a coadjoint pair on $P$ if the following conditions are satisfied.

(1) $\oplus: P \times P \rightarrow P$ is isotone.

(2) $\ominus: P \times P \rightarrow P$ is isotone on first variable and antitone on second variable.

(3) $a \leq b \oplus c$ iff $a \ominus c \leq b, a, b, c \in P$.

Definition 2 (see [12]). A structure $(L ; \vee, \wedge, \oplus, \ominus, 0,1)$ is called a coresiduated lattice if the following conditions are satisfied.

(1) $(L ; \vee, \wedge)$ is a bounded lattice, 0 is the smallest element, and 1 is the greatest element of $L$, respectively.

(2) $(\oplus, \ominus)$ is a coadjoint pair on $L$.

(3) $(L ; \oplus, 0)$ is a commutative monoid.

$\oplus$ is called a $t$-conorm operator on $L$, and $\ominus$ is called a fuzzy difference operator on $L$. One denotes CRL as the set of all coresiduated lattices.

Theorem 3 (see [12]). Suppose $(L ; \vee, \wedge, \oplus, \ominus, 0,1) \in C R L$; then

(1) $a \ominus 0=a$,

(2) $a \leq b$ iff $a \ominus b=0$,

(3) $a \oplus b=0$ iff $a=b=0$,

(4) $(a \ominus b) \ominus c=(a \ominus c) \ominus b=a \ominus(b \oplus c)$,

(5) $(a \oplus b) \ominus b \leq a \leq(a \ominus b) \oplus b$,

(6) $(a \oplus b) \ominus b \ominus a=(a \oplus b) \ominus a \ominus b=0$,

(7) $a \ominus(b \wedge c)=(a \ominus b) \vee(a \ominus c)$,

(8) $(a \vee b) \ominus c=(a \ominus c) \vee(b \ominus c)$,

(9) $(a \vee b) \ominus b=a \ominus b$,

(10) $a \ominus(a \wedge b)=a \ominus b$,

(11) $a \ominus(a \ominus b) \leq a \wedge b$,

(12) $(a \ominus b) \oplus b \geq a \vee b$,

(13) $(a \oplus c) \ominus(c \oplus b) \leq a \ominus b \leq(a \ominus c) \oplus(c \ominus b)$.

Definition 4 (see [15]). Suppose $L \in \mathrm{CRL}, I \subseteq L$; $I$ is an ideal of $L$ if one has the following:

(1) $0 \in I$.

(2) If $x \in I, y \in L$, and $y \leq x$, then $y \in I$.

(3) If $x \in I, y \in I$, then $x \oplus y \in I$.

$I$ is a real ideal of $L$ if $I \neq L$. A real ideal $I$ of $L$ is a prime ideal if

(4) $\forall x, y \in L$, if $x \wedge y \in I$, then $x \in I$ or $y \in I$. A real ideal $I$ of $L$ is a strong prime ideal if

(5) $\forall x, y \in L, x \ominus y \in I$ or $y \ominus x \in I$.

Theorem 5 (see [15]). Suppose $L \in C R L, I \subseteq L ; I$ is an ideal of $L$ if and only if one has the following:

(1) $0 \in I$.

(2) If $x \in I, y \ominus x \in I$, then $y \in I$.
Theorem 6 (see [15]). Suppose $L \in C R L$. I is a real ideal of $L$ and $A$ is a (strong) prime ideal of $L$; if $A \subseteq I$, then $I$ is a (strong) prime ideal of $L$.

Proposition 7 (see [15]). Consider $L \in C R L$; if I is a strong prime ideal of $L$, then $I$ is a prime ideal of $L$.

\section{Fuzzy Ideals of Coresiduated Lattices}

Definition 8. Suppose that $f$ is a fuzzy set on $L, L \in$ CRL. $f$ is a fuzzy ideal of $L$ if the following conditions are satisfied:

(P1) $f(0) \leq f(x), \forall x \in L$,

$(P 2) f(y) \leq f(y \ominus x) \vee f(x)$.

Throughout the rest of this paper, unless otherwise stated, $L$ always represents a coresiduated lattice and $f$ always represents a fuzzy set on $L$.

Proposition 9. Consider $L \in C R L$ and $f$ is a fuzzy ideal of $L$; then $f$ is isotone; that is,

$$
\text { (P3) } x \leq y \Rightarrow f(x) \leq f(y) \text {. }
$$

Proof. $\forall x, y \in L$, if $x \leq y$, then $x \ominus y=0$. By $(P 1)$ and (P2), it follows that

$$
f(x) \leq f(x \ominus y) \vee f(y)=f(0) \vee f(y)=f(y) .
$$

Theorem 10. Consider $L \in C R L$ and $f$ is a fuzzy ideal of $L$ if and only if

$$
(P 4) z \leq x \oplus y \Rightarrow f(z) \leq f(x) \vee f(y) .
$$

Proof.

Sufficiency. Consider $\forall x, y \in L, 0 \leq x \oplus x$; it follows by (P4) that

$$
f(0) \leq f(x) \vee f(x)=f(x) .
$$

So $(P 1)$ holds. From Theorem 3(5), $y \leq(y \ominus x) \oplus x$; it follows by $(P 4)$ that

$$
f(y) \leq f(y \ominus x) \vee f(x)
$$

So $(P 2)$ holds.

Necessity. If $z \leq x \oplus y$, then $z \ominus y \leq x$. Because $f$ is a fuzzy ideal of $L$, then it follows from $(P 3)$ that $f(z \ominus y) \leq f(x)$. According to $(P 2)$,

$$
f(z) \leq f(z \ominus y) \vee f(y) .
$$

Thus $f(z) \leq f(x) \vee f(y)$. So (P4) holds.

From Theorem 10, it is easy to get the following corollary. 
Corollary 11. Consider $L \in C R L$ and $f$ is a fuzzy set on $L$; then the following conditions are equivalent:

(1) $f$ is a fuzzy ideal of $L$; that is, (P1) and (P2) hold.

(2) $x \leq y \Rightarrow f(x) \leq f(y)$; that is, (P3) holds.

(3) $(z \ominus y) \ominus x=0 \Rightarrow f(z) \leq f(x) \vee f(y)$.

(4) $z \ominus(y \oplus x)=0 \Rightarrow f(z) \leq f(x) \vee f(y)$.

Corollary 12. Consider $L \in C R L$ and $f$ is a fuzzy ideal on $L$ if and only if

(P5) $z \leq x_{1} \oplus x_{2} \oplus \cdots \oplus x_{n} \Rightarrow f(z) \leq \max \left\{f\left(x_{1}\right), f\left(x_{2}\right), \ldots\right.$, $\left.f\left(x_{n}\right)\right\}, n \geq 2$.

Proof.

Sufficiency. If $n=2$, it is obviously valid. If $n>2$, let $z=0$ and $x_{i}=x, 1 \leq i \leq n, \forall x \in L$, from $0 \leq x \oplus x \oplus \cdots \oplus x$; then $f(0) \leq f(x)$.

If $z \leq x \oplus y$, let $x_{1}=x, x_{2}=y, x_{i}=0,2<i \leq n$; then $f(z) \leq f(x) \vee f(y) \vee f(0)=f(x) \vee f(y)$; that is, (P4) holds. Thus $f$ is a fuzzy ideal on $L$.

Necessity. Consider $n=2$; it is obviously valid. Suppose that it is valid if $n=k, k \geq 2$; that is,

$$
\begin{aligned}
z & \leq x_{1} \oplus x_{2} \oplus \cdots \oplus x_{k} \Longrightarrow \\
f(x) & \leq \max \left\{f\left(x_{1}\right), f\left(x_{2}\right), \ldots, f\left(x_{k}\right)\right\} .
\end{aligned}
$$

Then, put $n=k+1$; if $z \leq x_{1} \oplus x_{2} \oplus \cdots \oplus x_{k} \oplus x_{k+1}$, then

$$
z \ominus x_{k+1} \leq x_{1} \oplus x_{2} \oplus \cdots \oplus x_{k} \text {. }
$$

So

$$
f\left(z \ominus x_{k+1}\right) \leq \max \left\{f\left(x_{1}\right), f\left(x_{2}\right), \ldots, f\left(x_{k}\right)\right\} .
$$

Since $f$ is a fuzzy set on $L$, it is obtained that $f(z) \leq f(z \ominus$ $\left.x_{k+1}\right) \vee f\left(x_{k}\right)$. Therefore,

$$
f(z) \leq \max \left\{f\left(x_{1}\right), f\left(x_{2}\right), \ldots, f\left(x_{k+1}\right)\right\} .
$$

The proof is completed.

Theorem 13. Consider $L \in C R L$ and $f$ is a fuzzy ideal of $L$ if and only if

(1) $f$ is isotone; that is, (P3) holds;

(2) $(P 6) f(x \oplus y)=f(x) \vee f(y)$.

Proof.

Sufficiency. Suppose that (P3) and (P6) hold; it should be proved that (P4) holds. If $z \leq x \oplus y$, it follows by (P3) and (P6) that

$$
f(z) \leq f(x \oplus y)=f(x) \vee f(y) .
$$

So (P4) holds.

Necessity. If $f$ is a fuzzy ideal of $L$, it follows from Proposition 9 that $f$ is isotone. Since $x \leq x \oplus y, y \leq x \oplus y$, then $f(x) \leq f(x \oplus y), f(y) \leq f(x \oplus y)$. Thus $f(x) \vee f(y) \leq f(x \oplus y)$.
Moreover, $x \oplus y \leq x \oplus y$; it follows from (P4) that $f(x \oplus$ $y) \leq f(x) \vee f(y)$. Therefore, $f(x \oplus y)=f(x) \vee f(y)$. The proof is completed.

Let $f$ be a fuzzy set on the coresiduated lattice $L$; it is denoted that

$$
f_{\lambda}=\{x \in L \mid f(x) \leq \lambda\}, \quad \lambda \in[0,1] .
$$

Theorem 14. $f$ is a fuzzy ideal of $L$ if and only if, $\forall \lambda \in[0,1]$, $f_{\lambda}$ is either an empty set or an ideal of $L$.

Proof.

Sufficiency. $\forall x, y \in L$, suppose that $f(x)=\lambda_{0}$, and let $f_{\lambda_{0}}=$ $\left\{z \mid f(z) \leq \lambda_{0}\right\}$. It is obvious that $f_{\lambda_{0}}$ is not an empty set; then $f_{\lambda_{0}}$ is an ideal of $L$, so $0 \in f_{\lambda_{0}}$. Thus $f(0) \leq \lambda_{0}=f(x)$; that is, $(P 1)$ holds. Put $\lambda_{1}=f(x) \vee f(y \ominus x)$; then $f(x) \leq$ $\lambda_{1}, f(y \ominus x) \leq \lambda_{1}$; that is, $x, y \ominus x \in f_{\lambda_{1}}$. Because $f_{\lambda_{1}}$ is an ideal of $L$, then $y \in f_{\lambda_{1}}$; that is, $f(y) \leq \lambda_{1}=f(x) \vee f(y \ominus x)$; thus (P2) holds. Therefore, $f$ is a fuzzy ideal of $L$.

Necessity. Suppose that $f_{\lambda}$ is not an empty set; then $\exists x_{0} \in$ $f_{\lambda}$ s.t. $f\left(x_{0}\right) \leq \lambda$. Since $f$ is a fuzzy ideal of $L$, then $f(0) \leq$ $f\left(x_{0}\right) \leq \lambda$; that is, $0 \in f_{\lambda}$. Suppose $x, y \ominus x \in f_{\lambda}$; then $f(x) \leq$ $\lambda, f(y \ominus x) \leq \lambda$. Thus $f(y) \leq f(x) \vee f(y \ominus x) \leq \lambda$; that is, $y \in f_{\lambda}$. It follows from Theorem 5 that $f_{\lambda}$ is an ideal of $L$.

Corollary 15. Suppose that $f$ is a fuzzy ideal of $L$; then $\forall a \in L$,

$$
\Omega_{a}=\{x \in L \mid f(x) \leq f(a)\}
$$

is an ideal of $L$.

Proof. Since $f(a) \leq f(a)$, it is obtained that $a \in \Omega_{a}$; that is, $\Omega_{a} \neq \emptyset$. It follows from Theorem 14 that $\Omega_{a}$ is an ideal of $L$.

Corollary 16. Suppose that $f$ is a fuzzy ideal of $L$; then

$$
\Omega_{f}=\{x \in L \mid f(x)=f(0)\}
$$

is an ideal of $L$.

Proof. Since $f$ is a fuzzy ideal of $L$, then $f(0) \leq f(x), x \in L$. So

$$
\begin{aligned}
\Omega_{f} & =\{x \in L \mid f(x)=f(0)\} \\
& =\{x \in L \mid f(x) \leq f(0)\}=\Omega_{0}
\end{aligned}
$$

By Corollary 15, $\Omega_{0}$ is an ideal of $L$. Thus $\Omega_{f}$ is an ideal of $L$.

Theorem 17. $f$ is a fuzzy set on $L, a \in L$. If $f$ satisfies the conditions

(1) $f$ is isotone,

(2) $\forall x, y \in L, f(x) \leq f(a), f(y) \leq f(a) \Rightarrow f(x \oplus y) \leq$ $f(a)$,

then $\Omega_{a}$ is an ideal of $L$. 
Proof. Since $0 \leq a$ and $f$ is isotone, then $f(0) \leq f(a)$; that is, $0 \in \Omega_{a}$. If $x \in \Omega_{a}$ and $y \leq x$, then $f(y) \leq f(x) \leq f(a)$; that is, $y \in \Omega_{a}$. According to (2), $x, y \in \Omega_{a} \Rightarrow x \oplus y \in \Omega_{a}$. Therefore, $\Omega_{a}$ is an ideal of $L$.

\section{Fuzzy Prime Ideals and Fuzzy Strong Prime Ideals of Coresiduated Lattices}

Definition 18. Suppose that $f$ is a fuzzy ideal of $L$ and not a constant. $f$ is a fuzzy prime ideal of $L$ if the following conditions are satisfied:

(P7) $f(x \wedge y)=f(x) \wedge f(y), \forall x, y \in L$.

In this section, it is always assumed that $f$ is a fuzzy set on $L$ and not a constant.

Theorem 19. $f$ is a fuzzy prime ideal of $L$ if and only if one has the following: if $\emptyset \neq f_{\lambda} \varsubsetneqq L$, then $f_{\lambda}$ is a prime ideal of $L$.

Proof.

Sufficiency. Since $f$ is not a constant, then $\exists \lambda \in[0,1]$ s.t. $\emptyset \neq$ $f_{\lambda} \varsubsetneqq L$. So $f_{\lambda}$ is a prime ideal of $L$. If $f_{\lambda}$ is a prime ideal of $L$, then it is obviously an ideal of $L$. From Theorem $14, f$ is a fuzzy ideal of $L$. So $f$ is isotone. $\forall x, y \in L$, put $f(x \wedge y)=$ $\lambda_{0}$; then $x \wedge y \in f_{\lambda_{0}}$. Since $f_{\lambda_{0}}$ is a prime ideal, it follows from Definition 4 that $x \in f_{\lambda_{0}}$ or $y \in f_{\lambda_{0}}$. Let $x \in f_{\lambda_{0}}$; then $f(x) \leq \lambda_{0}=f(x \wedge y) \leq f(x) \wedge f(y)$. By $f(x) \wedge f(y) \leq f(x)$, $f(x \wedge y)=f(x) \wedge f(y)$; that is, $f$ is a fuzzy prime ideal of $L$.

Necessity. $\forall \lambda \in[0,1]$, if $x \wedge y \in f_{\lambda}$, then $f(x \wedge y) \leq \lambda$. Because $f$ is a fuzzy prime ideal of $L, f(x \wedge y)=f(x) \wedge f(y) \leq \lambda$. Thus $f(x) \leq \lambda$ or $f(y) \leq \lambda$; that is, $x \in f_{\lambda}$ or $y \in f_{\lambda}$. Therefore, $f_{\lambda}$ is a prime ideal of $L$.

Definition 20. Suppose that $f$ is a fuzzy ideal of $L$ and not a constant. $f$ is a fuzzy strong prime ideal of $L$ if the following conditions are satisfied:

(P8) $f(x \ominus y)=f(0)$ or $f(y \ominus x)=f(0), \forall x, y \in L$.

Theorem 21. $f$ is a fuzzy strong prime ideal of $L$ if and only if one has the following: if $\emptyset \neq f_{\lambda} \subsetneq L$, then $f_{\lambda}$ is a strong prime ideal of $L$.

Proof.

Sufficiency. Since $f$ is not a constant, then $\exists \lambda \in[0,1]$ s.t. $\emptyset \neq f_{\lambda} \varsubsetneqq L$. So $f_{\lambda}$ is a strong prime ideal of $L$. If $f_{\lambda}$ is a strong prime ideal of $L$, then it is obviously an ideal of $L$. From Theorem 14, $f$ is a fuzzy ideal of $L$. So $f$ is isotone. $\forall x, y \in L$, put $f(0)=\lambda_{0}$; then $0 \in f_{\lambda_{0}}$. Since $f_{\lambda_{0}}$ is a strong prime ideal, $x \ominus y \in f_{\lambda_{0}}$ or $y \ominus x \in f_{\lambda_{0}}$. Let $x \ominus y \in f_{\lambda_{0}}$; then $f(x \ominus y) \leq \lambda_{0}=f(0)$. Because $f$ is isotone, $f(0) \leq f(x \ominus y)$. It is obtained that $f$ is a fuzzy strong prime ideal of $L$.

Necessity. Since $f$ is a fuzzy strong prime ideal of $L$, it is obvious that $f$ is a fuzzy ideal of $L$. According to Theorem 14, $\forall \lambda \in[0,1]$, if $f_{\lambda}$ is an ideal of $L$, then $0 \in f_{\lambda}$; that is, $f(0) \leq \lambda$. Moreover, $f$ is a fuzzy strong prime ideal of $L$.
If $f(x \ominus y)=f(0)$, then $f(x \ominus y) \leq \lambda$; that is, $x \ominus y \in f_{\lambda}$. Therefore, $f_{\lambda}$ is a strong prime ideal of $L$.

Theorem 22. If $f$ is a fuzzy strong prime ideal of $L$ then $f$ is a fuzzy prime ideal of $L$.

Proof. $f$ is a fuzzy strong prime ideal of $L . \forall x, y \in L$, let $f(x \ominus$ $y)=f(0)$. It follows from Theorem 13,

$$
\begin{aligned}
& f((x \wedge y) \oplus(x \ominus y))=f(x \wedge y) \vee f(x \ominus y) \\
& \quad=f(x \wedge y) \vee f(0)=f(x \wedge y) .
\end{aligned}
$$

By Theorem 3(5) and (7),

$$
\begin{aligned}
(x \wedge y) \oplus(x \ominus y) & =(x \oplus(x \ominus y)) \wedge(y \oplus(x \ominus y)) \\
& \geq(x \oplus(x \ominus y)) \wedge x \geq(x \oplus 0) \wedge x \\
& =x .
\end{aligned}
$$

Since $f$ is isotone, then $f((x \wedge y) \oplus(x \ominus y)) \geq f(x) \geq f(x) \wedge$ $f(y)$. Thus $f(x \wedge y) \geq f(x) \wedge f(y)$. Obviously, $f(x \wedge y) \leq$ $f(x) \wedge f(y)$. So $f(x \wedge y)=f(x) \wedge f(y)$; that is, (P6) holds. Therefore $f$ is a fuzzy prime ideal of $L$.

Suppose that $I$ is a real ideal of $L$; put

$$
\chi_{I}= \begin{cases}0 & x \in I, \\ 1 & x \bar{\in} I .\end{cases}
$$

According to Theorem 14, $\chi_{I}$ is a fuzzy ideal and not a constant. By Theorems 19 and 21, we can get the following two theorems, respectively.

Theorem 23. I is a prime ideal of $L$ if and only if $\chi_{I}$ is a fuzzy prime ideal of $L$.

Theorem 24. $I$ is a strong prime ideal of $L$ if and only if $\chi_{I}$ is a fuzzy strong prime ideal of $L$.

\section{Fuzzy Ideals and Fuzzy Prime Ideals of Prelinear Coresiduated Lattices}

In a residuated lattice $L$, the condition

$$
(x \longrightarrow y) \vee(y \longrightarrow x)=1, \quad x, y \in L
$$

is called prelinearity axiom (see [1]). Taking account of coresiduated lattice as the dual algebra structure of residuated lattice, the definition of prelinear coresiduated lattice is as follows.

Definition 25 (see [15]). A coresiduated lattice $L$ is called a prelinear coresiduated lattice if, $\forall x, y \in L,(x \ominus y) \wedge(y \ominus x)=0$.

Theorem 26 (see [15]). Suppose that $L$ is a prelinear coresiduated lattice; $I$ is an ideal of $L$ and $a \in L \backslash I$; then there exists a prime ideal $J$ of $L$ such that $I \subseteq J$ and $a \bar{\epsilon} J$. 
Theorem 27. $L$ is a prelinear coresiduated lattice. $f$ is a fuzzy strong prime ideal of $L$ if and only if $f$ is a fuzzy prime ideal of L.

Proof. Necessity is obvious by Theorem 22 .

Sufficiency. $\forall x, y \in L$, since $L$ is a prelinear coresiduated lattice, then $(x \ominus y) \wedge(y \ominus x)=0$. Because $f$ is a fuzzy prime ideal of $L$,

$$
\begin{aligned}
& f(x \ominus y) \wedge f(y \ominus x)=f((x \ominus y) \wedge(x \ominus y)) \\
& \quad=f(0) .
\end{aligned}
$$

Thus $f(x \ominus y)=f(0)$ or $f(y \ominus x)=f(0)$.

Corollary 28. Suppose that $L$ is a prelinear coresiduated lattice and $f$ is a fuzzy ideal of $L$; then the following conditions are equivalent:

(1) $f$ is a fuzzy prime ideal of $L$.

(2) $f(x \wedge y)=f(0) \Rightarrow f(x)=0$ or $f(y)=0$.

(3) $f$ is a fuzzy strong prime ideal of $L$.

Proof. It is only proved that (1) $\Rightarrow(2)$ and (2) $\Rightarrow(3)$.

$(1) \Rightarrow(2)$ : Since $f$ is a fuzzy prime ideal of $L$, then $f(x \wedge$ $y)=f(x) \wedge f(y)$. Because $f(x \wedge y)=f(0), f(x) \wedge f(y)=0$. Thus $f(x)=0$ or $f(y)=0$.

$(2) \Rightarrow(3): L$ is a prelinear coresiduated lattice; then $(x \ominus$ $y) \wedge(y \ominus x)=0$. So $f((x \ominus y) \wedge(x \ominus y))=f(0)$. According to (2), it follows that $f(x \ominus y)=f(0)$ or $f(y \ominus x)=f(0)$.

Remark 29. According to Theorem 27, we do not distinguish between fuzzy strong prime ideal and fuzzy prime ideal in prelinear coresiduated lattices.

Theorem 30. Suppose that $L$ is a prelinear coresiduated lattice; $f$ is a fuzzy ideal and not a constant; then the following conditions are equivalent:

\section{(1) $L$ is a chain.}

(2) Any fuzzy ideal which is not a constant is a fuzzy prime ideal.

(3) Any fuzzy ideal such that $f(0)=0$ and not a constant is a fuzzy prime ideal.

(4) The fuzzy ideal $\chi_{\{0\}}$ is a fuzzy prime ideal.

Proof. (1) $\Rightarrow(2): \forall x, y \in L$, since $L$ is a chain, then $x \leq y$ or $y \leq x$. Thus $x \ominus y=0$ or $y \ominus x=0$. So $f(x \ominus y)=f(0)$ or $f(y \ominus x)=f(0)$. Therefore $f$ is a fuzzy prime ideal.

It is obvious that (2) $\Rightarrow(3)$ and (3) $\Rightarrow(4)$.

$(4) \Rightarrow(1): \chi_{\{0\}}$ is a fuzzy prime ideal; then $\forall x, y \in L$, $\chi_{\{0\}}(x \ominus y)=\chi_{\{0\}}(0)=0$ or $\chi_{\{0\}}(y \ominus x)=\chi_{\{0\}}(0)=0$. It is obtained that $x \ominus y \in\{0\}$ or $y \ominus x \in\{0\}$; that is, $x \ominus y=0$ or $y \ominus x=0$. So $x \leq y$ or $y \leq x$; that is, $L$ is a chain.

Theorem 31. Suppose that $L$ is a prelinear coresiduated lattice and $f$ is a fuzzy prime ideal. If $g$ is a fuzzy ideal and not a constant satisfying $g \leq f, g(0)=f(0)$, then $g$ is a fuzzy prime ideal.
Proof. Since $f$ is a fuzzy prime ideal, then, $\forall x, y \in L, f(x \ominus$ $y)=f(0)$ or $f(y \ominus x)=0$. Let $f(x \ominus y)=f(0)$. It follows from $g \leq f, g(0)=f(0)$ that

$$
g(x \ominus y) \leq f(x \ominus y)=f(0)=g(0) .
$$

Because $g$ is a fuzzy ideal, $g$ is isotone. Thus $g(0) \leq g(x \ominus y)$. So $g(x \ominus y)=g(0)$. Therefore, $g$ is a fuzzy strong prime ideal. The proof is completed.

Theorem 32. Suppose that $L$ is a prelinear coresiduated lattice; $f$ is a fuzzy prime ideal and $\alpha \in(f(0), 1]$; then $f \wedge \alpha$ is a fuzzy prime ideal of $L$ where

$$
(f \wedge \alpha)(x)=f(x) \wedge \alpha .
$$

Proof. Since $f$ is a fuzzy prime ideal, then $f$ is a fuzzy ideal. $\forall x, y, z \in L$, if $z \leq x \oplus y$, then $f(z) \leq f(x) \vee f(y)$. So

$$
\begin{aligned}
(f \wedge \alpha)(z) & =f(z) \wedge z \leq(f(x) \vee f(y)) \wedge \alpha \\
& =(f(x) \wedge \alpha) \vee(f(y) \wedge \alpha) \\
& =(f \wedge \alpha)(x) \vee(f \wedge \alpha)(y) .
\end{aligned}
$$

Thus $f \wedge \alpha$ is a fuzzy ideal. By $\alpha \in(f(0), 1]$, it is obtained that $f \wedge \alpha$ is not a constant and $(f \wedge \alpha)(0)=f(0) \wedge \alpha=f(0)$, $(f \wedge \alpha)(x)=f(x) \wedge \alpha \leq f(x)$. Since $f$ is a fuzzy prime ideal, then according to Theorem $31 f \wedge \alpha$ is a fuzzy prime ideal of $L$.

Theorem 33. Suppose that $L$ is a prelinear coresiduated lattice and $f$ is a fuzzy ideal and not a constant satisfying $f(0)>0$; then there exists a fuzzy prime ideal $g$ such that $g \leq f$.

Proof. Since $f$ is a fuzzy ideal and not a constant, it follows that $\exists a \in L$, s.t. $f(a) \neq f(0)$; that is, $a \bar{\epsilon} \Omega_{f}$. So $\Omega_{f}$ is a real ideal of $L$. It follows from Theorem 26 that there exists a prime ideal $I$ such that $\Omega_{f} \subseteq I$ and $a \bar{\in} I$. Thus $\chi_{I}$ is a fuzzy prime ideal. Put $\alpha=\inf \{f(x) \mid x \in L \backslash I\}$; then $\alpha \geq f(0)>0$. So it follows from Theorem 32 that $\chi_{I} \wedge \alpha$ is a fuzzy prime ideal. Let $g=\chi_{I} \wedge \alpha$; then $x \in I, g(x)=\left(\chi_{I}(x)\right) \wedge \alpha=0 \wedge \alpha=$ $0<f(0) \leq f(x) ; x \bar{\epsilon} I, g(x)=\left(\chi_{I}(x)\right) \wedge \alpha=1 \wedge \alpha=\alpha=$ $\inf \{f(x) \mid x \in L \backslash I\} \leq f(x)$. It follows from the above that $g \leq f$. The proof is completed.

The proof of the above theorem indicates how to obtain a fuzzy prime ideal derived from a fuzzy ideal in a prelinear coresiduated lattice.

\section{Conclusion}

Coresiduated lattice, as a dual algebra structure of residuated lattice, provides a new frame for logic algebras. It is a useful tool to characterize the intuitionistic fuzzy operators and plays a vital role in the theory basses of Triple I method of intuitionistic fuzzy reasoning. It is well known that ideal is an important part of algebra structure for various fuzzy logic semantics. From the point of view of fuzzy set, it can be characterized by using the notion of fuzzy ideal. In the paper, we mainly introduce the concepts of fuzzy ideal, fuzzy 
prime ideal, and fuzzy strong prime ideal to coresiduated lattices and derive some of their characterizations. We explore the relationship between fuzzy ideals and ideals in the coresiduated lattice as well. Moreover, we investigate the properties of fuzzy ideal and fuzzy (strong) prime ideal in prelinear coresiduated lattices.

The research on the relation between the proposed fuzzy ideals of coresiduated lattices and fuzzy ideals/filters of residuated lattices is worth doing. The detailed discussion about it will be given in our future work.

\section{Competing Interests}

The authors declare that they have no competing interests.

\section{Acknowledgments}

The authors acknowledge their supports from the Natural Science Foundation of China (nos. 61473336, 11401361, and 61572016) and the Fundamental Research Funds for the Central Universities (no. GK201403001).

\section{References}

[1] P. Hajek, Metamathematics of Fuzzy Logic, Kluwer Academic, Lodon, UK, 1998.

[2] F. Esteva and L. Godo, "Monoidal t-norm based logic: towards a logic for left-continuous t-norms," Fuzzy Sets and Systems, vol. 124, no. 3, pp. 271-288, 2001.

[3] R. L. Cignoli, I. M. D’Ottaviano, and D. Mundici, Algebraic Foundations of Many-Valued Reasoning, vol. 7, Kluwer Academic Publishers, Dordrecht, The Netherlands, 2000.

[4] C. C. Chang, "Algebraic analysis of many valued logics," Transactions of the American Mathematical Society, vol. 88, pp. 467490, 1958

[5] G. J. Wang, Introduction to Mathematical Logic and Resolution Principle, Science Press, Beijing, China, 2nd edition, 2006 (Chinese).

[6] D. W. Pei, "On equivalent forms of fuzzy logic systems NM and IMTL," Fuzzy Sets and Systems, vol. 138, no. 1, pp. 187-195, 2003.

[7] E. Turunen, "Boolean deductive systems of BL-algebras," Archive for Mathematical Logic, vol. 40, no. 6, pp. 467-473, 2001.

[8] M. Haveshki, A. B. Saeid, and E. Eslami, "Some types of filters in BL algebras," Soft Computing, vol. 10, no. 8, pp. 657-664, 2006.

[9] B. V. Gasse, G. Deschrijver, C. Cornelis, and E. E. Kerre, "Filters of residuated lattices and triangle algebras," Information Sciences, vol. 180, no. 16, pp. 3006-3020, 2010.

[10] L. Z. Liu and K. T. Li, "Boolean filters and positive implicative filters of residuated lattices," Information Sciences, vol. 177, no. 24, pp. 5725-5738, 2007.

[11] H. J. Zhou and B. Zhao, "Stone-like representation theorems and three-valued filters in $\mathrm{R}_{0}$-algebras (nilpotent minimum algebras)," Fuzzy Sets and Systems, vol. 162, no. 1, pp. 1-26, 2011.

[12] M. C. Zheng and G. J. Wang, "Co-residuated lattice with application," Fuzzy Systems and Mathematics, vol. 19, pp. 1-6, 2005 (Chinese).

[13] M. C. Zheng, Z. K. Shi, and Y. Liu, "Triple I method of approximate reasoning on Atanassov's intuitionistic fuzzy sets," International Journal of Approximate Reasoning, vol. 55, no. 6, pp. 1369-1382, 2014.
[14] Y. Liu and M. Zheng, "The dual triple I methods of FMT and IFMT," Mathematical Problems in Engineering, vol. 2014, Article ID 507401, 8 pages, 2014.

[15] M. C. Zheng, G. J. Wang, and Y. Liu, "Ideals and embedding theorem of co-residuated lattices," Journal of Shaanxi Normal University (Natural Science Edition), vol. 34, pp. 1-6, 2006 (Chinese).

[16] L. Z. Liu and K. T. Li, "Fuzzy filters of BL-algebras," Information Sciences, vol. 173, no. 1-3, pp. 141-154, 2005.

[17] L. Z. Liu and K. T. Li, "Fuzzy Boolean and positive implicative filters of BL-algebras," Fuzzy Sets and Systems, vol. 152, no. 2, pp. 333-348, 2005.

[18] Y. B. Jun, Y. Xu, and X. H. Zhang, "Fuzzy filters of MTLalgebras," Information Sciences, vol. 175, no. 1-2, pp. 120-138, 2005.

[19] Y. Q. Zhu and Y. Xu, "On filter theory of residuated lattices," Information Sciences, vol. 180, no. 19, pp. 3614-3632, 2010. 


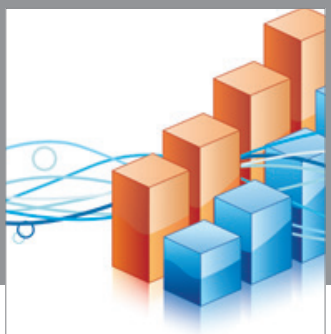

Advances in

Operations Research

vatem alat4

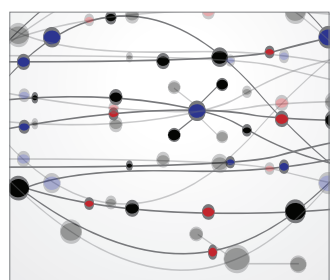

\section{The Scientific} World Journal
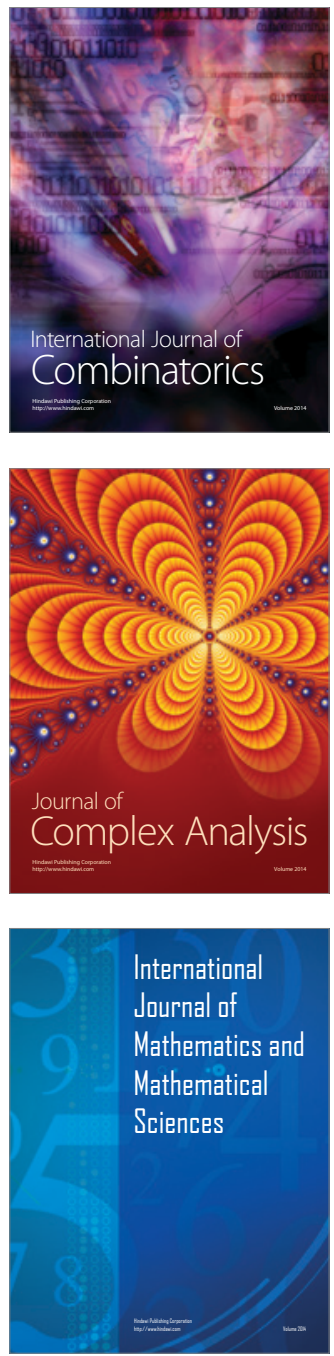
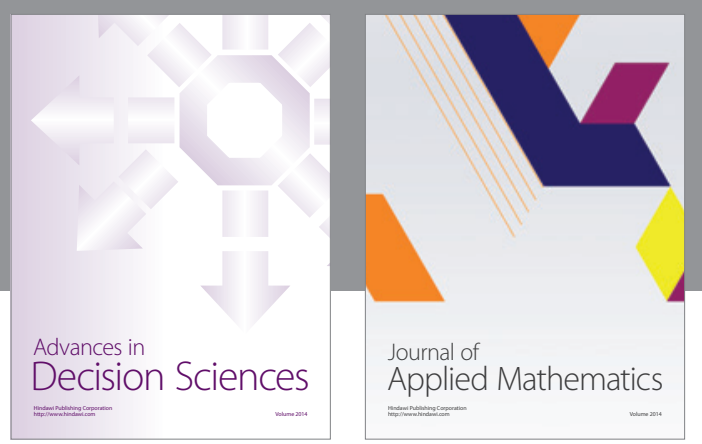

Algebra

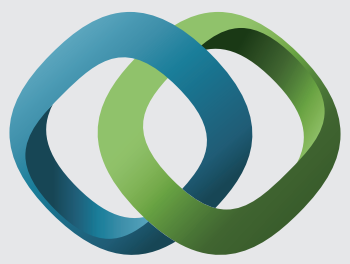

\section{Hindawi}

Submit your manuscripts at

http://www.hindawi.com
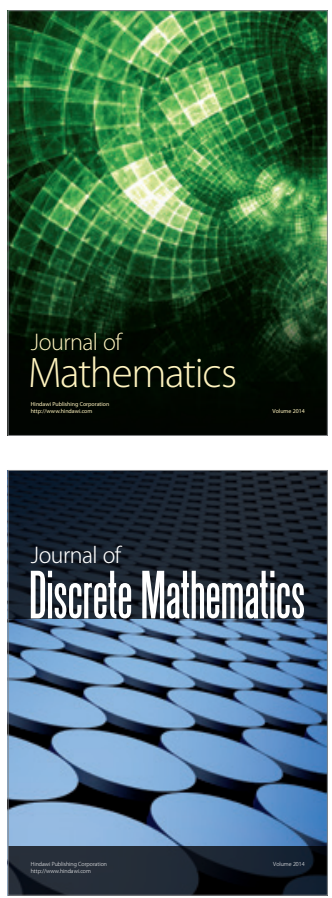

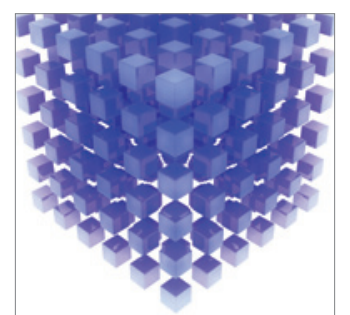

Mathematical Problems in Engineering
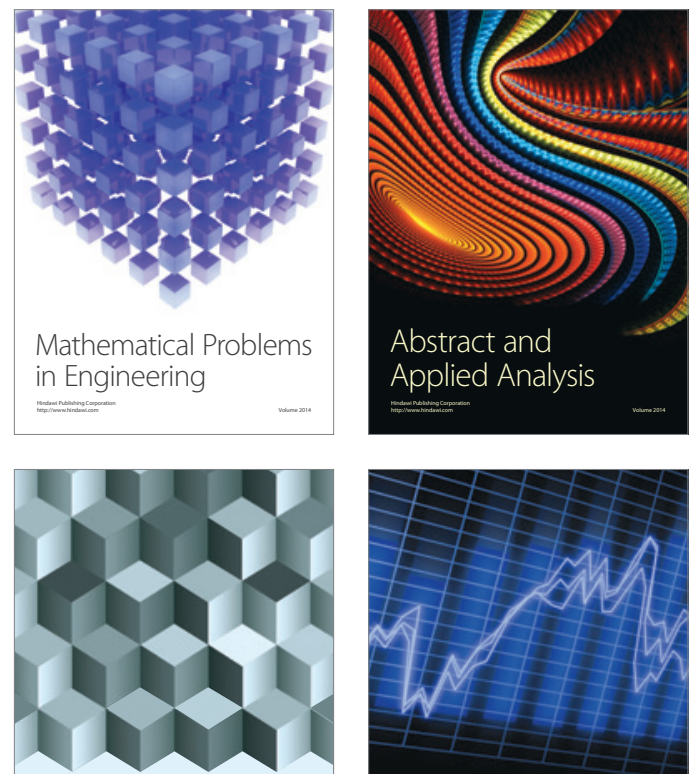

Journal of

Function Spaces

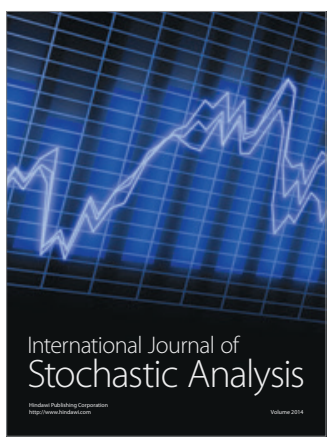

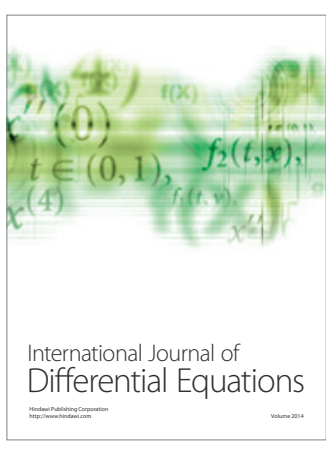
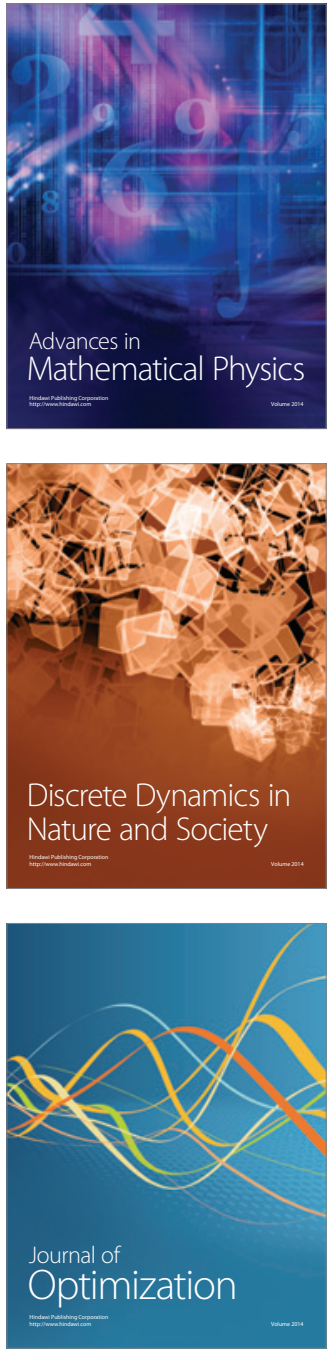